\title{
Darboux Iso-Geodesic Special Curve in Euclidean Space
}

\author{
M. M. Wageeda ${ }^{1}$, E. M. Solouma ${ }^{2} \&$ M. Bary ${ }^{1}$ \\ ${ }^{1}$ Department of Mathematics, Faculty of Science, Aswan University, Egypt \\ ${ }^{2}$ Department of Mathematics, Faculty of Science, Beni-Suef University, Egypt \\ Correspondence: M. Bary, Department of Mathematics, Faculty of Science, Aswan University, Egypt. E-mail: \\ m.bary96@gmail.com
}

Received: August 5, 2019

Accepted: August 27, 2019

Online Published: August 28, 2019

doi:10.5539/mas.v13n9p98

URL: https://doi.org/10.5539/mas.v13n9p98

\begin{abstract}
In this paper, by using Darboux frame we scrutinize the issues of reconstructing surfaces with given some unusual Smarandache curves in Euclidean 3-space, we make manifest the family of surfaces as a linear combination of the components of this frame and derive the necessary and sufficient conditions for coefficients to satisfy both the iso-geodesic and iso-parametric requirements.
\end{abstract}

Keywords: Smarandache curve, Darboux frame, Euclidean space-time

AMS Subject Classification (2010): Primary: 53A04; 53A05; 53C22; Secondary: 58E10.

\section{Introduction}

Geodesic on the surface may be an essential geometric part that holds a paramount part in the different applications. Geometrically, the geodesic on any surface may be an indispensable part simple curve on the surface such that for any two points on the curve a geodesic will be shortest path between them on the surface. Many geometric operations would initially associate to geodesics, they are also important in many applications, the method finds its applications in computer vision and image processing, such as in object segmentation, and multi-scale more analysis (P. N. Azariadis \& N. A. Aspragathos, 2001; R. J. Haw. 1985).

Also, the geodesics are important in the relativistic description of gravity. (Where Einstein's guideline about proportionality advises us that geodesics represent the paths of freely falling particles in a given space, the geodesics principle states that the free trajectories additionally the geodesics of space, and therefore has not to be set as an independent equation).

Also, the geodesics are important in architecture; a few exceptional curves bring decent properties As far as structural purpose Furthermore manufacturing expense. For instance, may be planar curves in perpendicular planes, which might be utilized similarly as help components (R. Brond et al., 1994; V. Caselles et al., 1997; F. Karakus \& Y. Yayli. 2016; C. Y. Li et al., 2001; G. J. Wang et al., 2004).

In this paper, by using Smarandache curve we will study what is the problem of the special curves which it is obtained with Darboux frame how to describe those surfaces that possess this curve as an iso-geodesic in three-dimension Euclidean space? and we will give some preliminary particulars and facts about this special curves in three- dimension Euclidean space, and define the iso-geodesic curve and we will study progressive surfaces as a linear combination of the Darboux frame of the presented curve, and derive necessary and sufficient conditions on marching scale functions to satisfy both iso-geodesic and Smarandache requirements.

\section{Preliminaries}

Let we are given a spatial parametric curve $C: \Upsilon(\varsigma)=(\mathrm{x}(\varsigma) ; \mathrm{y}(\varsigma) ; \mathrm{z}(\varsigma)) ; \quad 0 \leq \varsigma \leq \mathrm{L}$ and $\{\mathrm{T}(\varsigma), \mathrm{N}(\varsigma), \mathrm{B}(\varsigma)\}$ are seret-ferrent, where $\Upsilon(\varsigma)$ the arbitrarily parametrized geodesic, $\mathrm{T}(\zeta)$ is the unit tangent vector, $\mathrm{N}(\zeta)$ is the Frenet principal normal vector, and $\mathrm{B}(\varsigma)$ is the Frenet binormal vector.

Let $\{\mathrm{T}(\varsigma), \mu(\varsigma), \xi(\varsigma)\}$ the Darboux frame, where $\{\mathrm{T}(\varsigma), \mu(\varsigma), \xi(\varsigma)\}$ the $\{$ unit tangent, unit normal, unit vector\} respectively (O. Bektas. \& S. Yuce. 2013), then the relations between this frame and the Frenet frame can be described as the subsequent: 


$$
\left[\begin{array}{l}
T(\varsigma) \\
\mu(\varsigma) \\
\xi(\varsigma)
\end{array}\right]=\left[\begin{array}{ccc}
1 & 0 & 0 \\
0 & \cos \theta & \sin \theta \\
0 & -\sin \theta & \cos \theta
\end{array}\right]\left[\begin{array}{l}
T(\varsigma) \\
N(\varsigma) \\
B(\varsigma)
\end{array}\right]
$$

From the differential and applying the Frenet Serret formulas we get the subsequent.

$$
\left\{\begin{array}{c}
\mathrm{T}(\mathrm{s})=\mathrm{K}_{\xi} \xi(\varsigma)-\mathrm{K}_{\mu} \mu(\mathrm{s}) \\
\xi(\varsigma)=-\mathrm{K}_{\xi} \mathrm{T}(\mathrm{s})+\tau \mu(\varsigma) \\
\mu(\varsigma)=\mathrm{K}_{\mu} \mathrm{T}(\mathrm{s})-\tau \xi(\varsigma)
\end{array}\right.
$$

Where $\mathrm{K}$ a curvature, $\tau$ a geodesic torsion, $\mathrm{K}_{\xi}=\mathrm{K} \cos \theta$ a geodesic curvature and $\mathrm{K}_{\mu}=\mathrm{K} \sin \theta$ is the normal curvature of this curve.

There is a condition if and only if this condition is met we know the curve $\mathrm{C}$ : $\mathrm{Y}(\varsigma)$ will be a geodesic on the surface $\psi(\varsigma, \mathrm{v})$, if the principal normal $\mu(\varsigma)$ to the curve $\mathrm{C}: \mathrm{Y}(\varsigma)$ and the normal vector $\mathrm{n}(\varsigma, \mathrm{v})$ to the surface $\psi(\varsigma, \mathrm{v})$ are parallel to each other at any point on the curve $\mathrm{C}: \mathrm{Y}(\varsigma)$, then the curve $\mathrm{C}$ : $\mathrm{Y}(\varsigma)$ will be a geodesic on the surface $\psi(\varsigma, \mathrm{v})$. And the curve $C: \Upsilon(\zeta)$ is an iso-parametric curve if the parameteres $\{\varsigma$ or $v\}$ are constant parameter value, i.e $\psi\left(\varsigma_{0}, v\right)=\Upsilon(\varsigma)$ or $\psi\left(\varsigma, v_{0}\right)=\Upsilon(\varsigma)$.

And the curve $C: \Upsilon(\varsigma)$ is an iso-geodesic of a surface $\psi(\varsigma, v)$ if it is both a geodesic and an iso-parametric curve on the surface. In other words, let can be taking the form

$$
n\left(\varsigma, v_{0}\right)=\Phi_{1}\left(\varsigma, V_{0}\right) T(\varsigma)+\Phi_{2}\left(s, V_{0}\right) N(\varsigma)+\Phi_{3}\left(\varsigma, V_{0}\right) B(\varsigma)
$$

if $n\left(\varsigma, v_{0}\right) / / N(\varsigma)$ where $n(\varsigma, v)$ is the normal vector of the curve and $N(\varsigma)$ is the Frenet principal normal vector on the surface, then we can consider that $C: \Upsilon(\varsigma)$ is an iso-geodesic on the surface, i.e. $\Phi_{2}\left(\varsigma, V_{0}\right) \neq 0$.

Suppose that $\mathrm{Y}(\varsigma)$ is a denote the unit speed regular curve in three-Dimension Euclidean space and the $\{\mathrm{TO}(\varsigma)$,

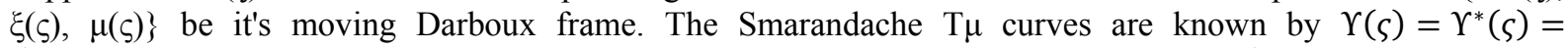
$\frac{1}{\sqrt{2}}(\mathrm{~T}(\varsigma)+\mu(\varsigma))$. And Smarandache $T \xi$ curves are known by $\Upsilon(\varsigma)=\Upsilon^{*}(\varsigma)=\frac{1}{\sqrt{2}}(\mathrm{~T}(\varsigma)+\xi(\varsigma))$. And Smarandache $\mu \xi$ curves are known by $\Upsilon(\varsigma)=\Upsilon^{*}(\varsigma)=\frac{1}{\sqrt{2}}(\xi(\varsigma)+\mu(\varsigma))$. And Smarandache $T \xi \mu$ curves are known by $\Upsilon(\varsigma)=\Upsilon^{*}(\varsigma)=\frac{1}{\sqrt{3}}(\mathrm{~T}(\varsigma)+\xi(\varsigma)+\mu(\varsigma))$.

\section{Surfaces with Common Smarandache Iso-Geodesic Curve}

Let the parametric surface $\psi(\varsigma, v):\left[L_{1}, L_{2}\right] \times\left[V_{1}, V_{2}\right] \rightarrow \Re^{3}$ is known stablished on the given curve $\Upsilon(\varsigma)$ and the local coordinate frame known by $T(\varsigma), \xi(\varsigma)$ and $\mu(\varsigma)$ as the subsequent:

$$
\begin{gathered}
\psi(\varsigma, v)=\Upsilon(\varsigma)+\{\mathrm{x}(\varsigma) \mathrm{T}(\varsigma)+\mathrm{Y}(\varsigma, \mathrm{v}) \xi(\varsigma)+\mathrm{Z}(\varsigma, \mathrm{v}) \mu(\varsigma)\} . \\
; L_{1} \leq \varsigma \leq L_{2}, V_{1} \leq v \leq V_{2}
\end{gathered}
$$

Where $X(\varsigma, v), Y(\varsigma, v)$ and $Z(\varsigma, v)$ are $C^{1}$ functions, and $X(\varsigma, v)$ the extension-like, $Y(\varsigma, v)$ exion-like and $Z(\varsigma, v)$ retortion-like influences by the point unit in the time $v$, starting from $\Upsilon(\varsigma)$ and $\{T(\varsigma), \xi(\varsigma), \mu(\varsigma)\}$ is the Darboux frame associated with the curve $\Upsilon(\varsigma)$. The normal vector $n(\varsigma, v)$ can be computed by taking the cross product of the partial differentials as the subsequent:

$$
\mathrm{n}(\mathrm{s}, \mathrm{v})=\frac{\partial \psi(\varsigma, \mathrm{v})}{\partial \varsigma} \times \frac{\partial \psi(\varsigma, \mathrm{v})}{\partial \mathrm{v}}
$$

Let the parameter $V_{1} \leq v \leq V_{2}, L_{1} \leq \varsigma \leq L_{2}$ such that $\psi\left(\varsigma, v_{0}\right)=\Upsilon(\varsigma)$, and the principal normal to the curve and the normal vector to the surface are parallel, then the our goal and important purpose in this work is to discover carefully the most important and adquate conditions that make some special Smarandache curves of the unit space curve $\Upsilon(\varsigma)$ on the surface $\psi(\varsigma, v)$ is an Iso-geodesic.

\subsection{Surfaces with Common $\boldsymbol{T} \boldsymbol{\mu}$ Curve}

Theorem 3.1. The Smarandache $T \mu$ of the curve $\Upsilon_{1}(\varsigma)$ is an iso-geodesic on a surface $\psi(\varsigma, v)$ if and only if this the subsequent condition is satisfed:

$$
\begin{array}{ll}
\text { 1. } & x\left(\varsigma, v_{0}\right)=y\left(\varsigma, v_{0}\right)=z\left(\varsigma, v_{0}\right)=0 \\
\text { 2. } & \frac{\partial z\left(\varsigma, v_{0}\right)}{\partial v}=\cot (\theta) \frac{\partial y\left(\varsigma, v_{0}\right)}{\partial v} \\
\text { 3. } & \frac{\partial\left(\varsigma, v_{0}\right)}{\partial v} \neq-\frac{\partial y\left(\varsigma, v_{0}\right)}{\partial v} \\
\text { 4. } & \mathrm{K}_{\xi}=\cot (\theta) \mathrm{K}_{\mu} \\
\text { 5. } & \mathrm{K}_{\mu} \neq 0 .
\end{array}
$$


proof: - Let $\Upsilon_{1}(\varsigma)$ be a Smarandache $T \mu$ curve on surface $\psi(\varsigma, v)$. From (3) we find the parametric surface is known by a Smarandache $T \mu$ as the subsequent:

$$
\psi(\varsigma, v)=\frac{1}{\sqrt{2}}(T(\varsigma)+\mu(\varsigma))+[x(\varsigma, v) T(\varsigma)+y(\varsigma, v) \mu(\varsigma)+z(\varsigma, v) \xi(\varsigma)]
$$

Ever since a spatial Smarandache curve of $T \mu$ is an iso-parametric curve on the surface $\psi(\varsigma, v)$, there dwell a parameter $v=v_{0} \in\left[V_{1}, V_{2}\right]$ such that $\psi(\varsigma, v)=\frac{1}{\sqrt{2}}(T(\varsigma)+\mu(\varsigma))$ and $\varsigma \in\left[L_{1}, L_{2}\right]$ then

$$
\mathrm{x}\left(\mathrm{s}, \mathrm{v}_{0}\right)=\mathrm{y}\left(\mathrm{s}, \mathrm{v}_{0}\right)=\mathrm{z}\left(\mathrm{s}, \mathrm{v}_{0}\right)=0
$$

From (4) the normal vector can be expressed as

Where

$$
n\left(\varsigma, v_{0}\right)=\alpha\left(\varsigma, v_{0}\right) T(\varsigma)+\beta\left(\varsigma, v_{0}\right) \mu(\varsigma)+\gamma\left(\varsigma, v_{0}\right) \xi(\varsigma),
$$

$$
\left\{\begin{array}{c}
\alpha\left(\varsigma, v_{0}\right)=\frac{\sqrt{2}}{2}\left(K_{\xi} \frac{\partial y\left(\varsigma, v_{0}\right)}{\partial v}-K_{\mu} \frac{\partial z\left(\varsigma, v_{0}\right)}{\partial v}\right) \\
\beta\left(\varsigma, v_{0}\right)=-\frac{\sqrt{2}}{2}\left(K_{\xi} \frac{\partial x\left(\varsigma, v_{0}\right)}{\partial v}+K_{\mu} \frac{\partial z\left(\varsigma, v_{0}\right)}{\partial v}\right) \\
\gamma\left(\varsigma, v_{0}\right)=\frac{\sqrt{2}}{2} K_{\mu}\left(\frac{\partial x\left(\varsigma, v_{0}\right)}{\partial v}+\frac{\partial y\left(\varsigma, v_{0}\right)}{\partial v}\right) .
\end{array}\right.
$$

From equation (1), (2) and (6) we find:

$$
n\left(\varsigma, v_{0}\right)=\Phi_{1}\left(\varsigma, v_{0}\right) T(\varsigma)+\Phi_{2}\left(\varsigma, v_{0}\right) N(\varsigma)+\Phi_{3}\left(\varsigma, v_{0}\right) B(\varsigma)
$$

Where

$$
\left\{\begin{array}{c}
\Phi_{1}\left(\varsigma, \mathrm{v}_{0}\right)=\alpha\left(\varsigma, \mathrm{v}_{0}\right)=0, \\
\Phi_{2}\left(\varsigma, \mathrm{v}_{0}\right)=\beta\left(\varsigma, \mathrm{v}_{0}\right) \cos (\theta)-\gamma\left(\varsigma, \mathrm{v}_{0}\right) \sin (\theta) \neq 0, \\
\Phi_{3}\left(\varsigma, \mathrm{v}_{0}\right)=\beta\left(\varsigma, \mathrm{v}_{0}\right) \sin (\theta)+\gamma\left(\varsigma, \mathrm{v}_{0}\right) \cos (\theta)=0 .
\end{array}\right.
$$

From (6) and (7) we find $\Phi_{1}\left(\varsigma, v_{0}\right)=0$ and $\Phi_{3}\left(\varsigma, v_{0}\right)=0$ if and only if these the subsequent conditions are satisfied

$$
\left\{\begin{aligned}
(1) \frac{\partial z\left(\varsigma, v_{0}\right)}{\partial v} & =\cot (\theta) \frac{\partial y\left(\varsigma, v_{0}\right)}{\partial v} \\
(2) \mathrm{K}_{\xi} & =\cot (\theta) \mathrm{K}_{\mu} .
\end{aligned}\right.
$$

Then $\Phi_{2} \neq 0$ is given by

$$
\frac{1}{\sqrt{2}} \mu(\varsigma) \cos (\theta)\left(\frac{\partial x\left(\varsigma, v_{0}\right)}{\partial v}+\frac{\partial y\left(\varsigma, v_{0}\right)}{\partial v}\right) \neq 0
$$

Then we have,

$$
\left\{\begin{array}{c}
(1) \frac{\partial x\left(\varsigma, v_{0}\right)}{\partial v} \neq-\frac{\partial y\left(\varsigma, v_{0}\right)}{\partial v} \\
(2) \mathrm{K}_{\mu} \neq 0 .
\end{array}\right.
$$

Then the Smarandache $T \mu$ of the curve is an iso-geodesic. From (5), (8) and (9) we have discovered the most important and adequate conditions for the $\psi\left(\zeta_{,}, v_{-} 0\right)$ have the spatial Smarandache $T \mu$ curve of the curve is an iso-geodesic.

\subsection{Surfaces with Common $\boldsymbol{T} \xi$ Curve}

Theorem 3.2. The Smarandache $T \xi$ of the curve $\Upsilon_{2}(\varsigma)$ is an iso-geodesic on a surface $\psi(\varsigma, v)$ if and only if this the subsequent condition is satisfed:

1. $x\left(s, v_{0}\right)=y\left(\varsigma, v_{0}\right)=z\left(\varsigma, v_{0}\right)=0$

2. $\frac{\partial y\left(\varsigma, v_{0}\right)}{\partial v}=\tan (\theta) \frac{\partial z\left(\varsigma, v_{0}\right)}{\partial v}$

3. $\frac{\partial x\left(\varsigma, v_{0}\right)}{\partial v} \neq-\frac{\partial z\left(\varsigma, v_{0}\right)}{\partial v}$

4. $\mathrm{K}_{\mu}=\tan (\theta) \mathrm{K}_{\xi}$

5. $\mathrm{K}_{\xi} \neq 0$.

Proof: Let $\Upsilon_{2}(\varsigma)$ be a Smarandache $T \xi$ curve on surface $\psi(\varsigma, v)$. From (3) we find the parametric surface is defined by a given Smarandache $T \xi$ as the subsequent:

$$
\psi(\varsigma, v)=\frac{1}{\sqrt{2}}(T(\varsigma)+\xi(\varsigma))+[x(\varsigma, v) T(\varsigma)+y(\varsigma, v) \mu(\varsigma)+z(\varsigma, v) \xi(\varsigma)]
$$


Even since Smarandache curve of $T \xi$ is an iso-parametric curve on the surface $\psi(\varsigma, v)$, there dwell a parameter $\mathrm{v}=\mathrm{v}_{0} \in\left[\mathrm{V}_{1}, \mathrm{~V}_{2}\right]$ such that $\psi(\mathrm{s}, \mathrm{v})=\frac{1}{\sqrt{2}}(\mathrm{~T}(\varsigma)+\xi(\varsigma))$ and $\varsigma \in\left[\mathrm{L}_{1}, \mathrm{~L}_{2}\right]$ then

$$
x\left(\varsigma, v_{0}\right)=y\left(\varsigma, v_{0}\right)=z\left(\varsigma, v_{0}\right)=0
$$

From (4) the normal vector can be expressed as

Where

$$
n\left(\varsigma, v_{0}\right)=\alpha\left(\varsigma, v_{0}\right) T(\varsigma)+\beta\left(\varsigma, v_{0}\right) \mu(\varsigma)+\gamma\left(\varsigma, v_{0}\right) \xi(\varsigma),
$$

$$
\left\{\begin{array}{l}
\alpha\left(\varsigma, \mathrm{v}_{0}\right)=\frac{\sqrt{2}}{2}\left(\mathrm{~K}_{\xi} \frac{\partial \mathrm{y}\left(\varsigma, \mathrm{v}_{0}\right)}{\partial \mathrm{v}}-\mathrm{K}_{\mu} \frac{\partial \mathrm{z}\left(\varsigma, \mathrm{v}_{0}\right)}{\partial \mathrm{v}}\right), \\
\beta\left(\varsigma, \mathrm{v}_{0}\right)=-\frac{\sqrt{2}}{2} \mathrm{~K}_{\xi}\left(\frac{\partial \mathrm{x}\left(\varsigma, \mathrm{v}_{0}\right)}{\partial \mathrm{v}}+\frac{\partial \mathrm{z}\left(\varsigma, \mathrm{v}_{0}\right)}{\partial \mathrm{v}}\right), \\
\gamma\left(\varsigma, \mathrm{v}_{0}\right)=\frac{\sqrt{2}}{2}\left(\mathrm{~K}_{\mu} \frac{\partial \mathrm{x}\left(\varsigma, \mathrm{v}_{0}\right)}{\partial \mathrm{v}}+\mathrm{K}_{\xi} \frac{\partial \mathrm{y}\left(\varsigma, \mathrm{v}_{0}\right)}{\partial \mathrm{v}}\right) .
\end{array}\right.
$$

From equation (1), (2) and (11) we find:

$$
n\left(\varsigma, v_{0}\right)=\Phi_{1}\left(\varsigma, v_{0}\right) T(\varsigma)+\Phi_{2}\left(\varsigma, v_{0}\right) N(\varsigma)+\Phi_{3}\left(\varsigma, v_{0}\right) B(\varsigma)
$$

Where

$$
\left\{\begin{array}{c}
\Phi_{1}\left(\varsigma, \mathrm{v}_{0}\right)=\alpha\left(\varsigma, \mathrm{v}_{0}\right)=0, \\
\Phi_{2}\left(\varsigma, \mathrm{v}_{0}\right)=\beta\left(\varsigma, \mathrm{v}_{0}\right) \cos (\theta)-\gamma\left(\varsigma, \mathrm{v}_{0}\right) \sin (\theta) \neq 0, \\
\Phi_{3}\left(\varsigma, \mathrm{v}_{0}\right)=\beta\left(\varsigma, \mathrm{v}_{0}\right) \sin (\theta)+\gamma\left(\varsigma, \mathrm{v}_{0}\right) \cos (\theta)=0 .
\end{array}\right.
$$

From (11) and (12) we find $\Phi_{1}\left(\varsigma, v_{0}\right)=0 \quad$ and $\quad \Phi_{3}\left(\varsigma, v_{0}\right)=0$ if the subsequent conditions are satisfies

$$
\frac{\partial y\left(\zeta, v_{0}\right)}{\partial v}=\tan (\theta) \frac{\partial z\left(\zeta, v_{0}\right)}{\partial v}, \quad \mathrm{~K}_{\mu}=\tan (\theta) \mathrm{K}_{\xi} .
$$

From $\Phi_{2} \neq 0$ and by using (13) we have

$$
\frac{-1}{\sqrt{2}} \mathrm{~K}_{\xi} \sec (\theta)\left(\frac{\partial x\left(\varsigma, v_{0}\right)}{\partial v}+\frac{\partial z\left(\varsigma, v_{0}\right)}{\partial v}\right) \neq 0
$$

Then we find,

$$
\mathrm{K}_{\xi} \neq 0, \quad \frac{\partial x\left(\varsigma, v_{0}\right)}{\partial v}+\frac{\partial z\left(\varsigma, v_{0}\right)}{\partial v} \neq 0
$$

Then the $T \xi$ is an iso-geodesic. Using (10), (13) and (14) we have discovered the most important and adequate conditions for the $\psi\left(\varsigma, v_{-} 0\right)$ have the spatial Smarandache $T \xi$ curve of the curve is an iso-geodesic.

\subsection{Surfaces with Common $\boldsymbol{\mu} \xi$ Curve}

Theorem 3.3. The Smarandache $\mu \xi$ of the curve $\Upsilon_{3}(\varsigma)$ is an iso-geodesic on a surface $\psi(\varsigma, v)$ if and only if these the subsequent conditions are satisfied:

1. $x\left(\varsigma, v_{0}\right)=y\left(\varsigma, v_{0}\right)=z\left(\varsigma, v_{0}\right)=0$,

2. $\frac{\partial z\left(\varsigma, v_{0}\right)}{\partial v}=\cot (\theta) \frac{\partial y\left(\varsigma, v_{0}\right)}{\partial v}$

3. $\frac{\partial y\left(\varsigma, v_{0}\right)}{\partial v} \neq 0$

4. $\mathrm{K}_{\mu}+\mathrm{K}_{\xi} \neq 0$.

Proof: Let $\Upsilon_{3}(\varsigma)$ be a Smarandache $\mu \xi$ curve on surface $\psi(\varsigma, v)$. From (3) we find the parametric surface is defined by a given Smarandache $\mu \xi$ as the subsequent:

$$
\psi(\varsigma, v)=\frac{1}{\sqrt{2}}(\mu(\varsigma)+\xi(\varsigma))+[x(\varsigma, v) T(\varsigma)+y(\varsigma, v) \mu(\varsigma)+z(\varsigma, v) \xi(\varsigma)]
$$

Even since Smarandache curve of $\mu \xi$ is an iso-parametric curve on the surface $\psi(\varsigma, v)$, there dwell a parameter $v=v_{0} \in\left[V_{1}, V_{2}\right]$ such that $\psi(\varsigma, v)=\frac{1}{\sqrt{2}}(\mu(\varsigma)+\xi(\varsigma))$ and $\varsigma \in\left[L_{1}, L_{2}\right]$ then

$$
\mathrm{x}\left(\mathrm{s}, \mathrm{v}_{0}\right)=\mathrm{y}\left(\mathrm{s}, \mathrm{v}_{0}\right)=\mathrm{z}\left(\mathrm{s}, \mathrm{v}_{0}\right)=0
$$

From (4) the normal vector can be expressed as

$$
n\left(\varsigma, v_{0}\right)=\alpha\left(\varsigma, v_{0}\right) T(\varsigma)+\beta\left(\varsigma, v_{0}\right) \mu(\varsigma)+\gamma\left(\varsigma, v_{0}\right) \xi(\varsigma) .
$$

Where 


$$
\left\{\begin{array}{c}
\alpha\left(\varsigma, v_{0}\right)=0 \\
\beta\left(\varsigma, v_{0}\right)=-\frac{\sqrt{2}}{2}\left(K_{\mu}+K_{\xi}\right) \frac{\partial z\left(\varsigma, v_{0}\right)}{\partial v}, \\
\gamma\left(\varsigma, v_{0}\right)=\frac{\sqrt{2}}{2}\left(K_{\mu}+K_{\xi}\right) \frac{\partial y\left(\varsigma, v_{0}\right)}{\partial v} .
\end{array}\right.
$$

From equation (1), (2) and (16) we find:

$$
n\left(\varsigma, v_{0}\right)=\Phi_{1}\left(\varsigma, v_{0}\right) T(\varsigma)+\Phi_{2}\left(\varsigma, v_{0}\right) N(\varsigma)+\Phi_{3}\left(\varsigma, v_{0}\right) B(\varsigma)
$$

Where

$$
\left\{\begin{array}{c}
\Phi_{1}\left(\varsigma, \mathrm{v}_{0}\right)=\alpha\left(\varsigma, \mathrm{v}_{0}\right)=0, \\
\Phi_{2}\left(\varsigma, \mathrm{v}_{0}\right)=\beta\left(\varsigma, \mathrm{v}_{0}\right) \cos (\theta)-\gamma\left(\varsigma, \mathrm{v}_{0}\right) \sin (\theta) \neq 0, \\
\Phi_{3}\left(\varsigma, \mathrm{v}_{0}\right)=\beta\left(\varsigma, \mathrm{v}_{0}\right) \sin (\theta)+\gamma\left(\varsigma, \mathrm{v}_{0}\right) \cos (\theta)=0 .
\end{array}\right.
$$

From (16) and (17) we find $\Phi_{3}\left(\varsigma, v_{0}\right)=0$ we get,

$$
\frac{\partial z\left(\varsigma, v_{0}\right)}{\partial v}=\cot (\theta) \frac{\partial y\left(\varsigma, v_{0}\right)}{\partial v} .
$$

From $\Phi_{2} \neq 0$ and by using (18) we have

$$
\frac{-1}{\sqrt{2}}\left(\mathrm{~K}_{\mu}+\mathrm{K}_{\xi}\right) \csc (\theta) \frac{\partial y\left(\varsigma, v_{0}\right)}{\partial v} \neq 0
$$

Then we have,

$$
\frac{\partial y\left(s, v_{0}\right)}{\partial v} \neq 0
$$

And

$$
\left(\mathrm{K}_{\mu}+\mathrm{K}_{\xi}\right) \neq 0
$$

Then from (15), (18), (19) and (20) we have discovered the most important and adequate conditions for the $\psi\left(\zeta, v_{-} 0\right)$ have the spatial Smarandache $\mu \xi$ curve of the curve is an iso-geodesic.

\subsection{Surfaces with Common $\mathbf{T} \boldsymbol{\mu} \xi$ Curve}

Theorem3.4. The Smarandache $T \mu \xi$ curve of the curve $\Upsilon_{4}(\varsigma)$ is an iso-geodesic on a surface $\psi(\varsigma, v)$ if and only if these the subsequent conditions are satisfied:

1. $x\left(\varsigma, v_{0}\right)=y\left(\varsigma, v_{0}\right)=z\left(\varsigma, v_{0}\right)=0$

2. $\frac{\partial y\left(\varsigma, v_{0}\right)}{\partial v}=\frac{\mathrm{K}_{\mu}}{\mathrm{K}_{\xi}} \frac{\partial z\left(\varsigma, v_{0}\right)}{\partial v}$

3. $\mathrm{K}_{\xi}=\tan (\theta) \mathrm{K}_{\mu}$

4. $\frac{\partial x\left(\varsigma, v_{0}\right)}{\partial v} \neq-(1+\tan (\theta)) \frac{\partial z\left(s, v_{0}\right)}{\partial v}$

5. $\mathrm{K}_{\mu} \neq 0$.

Proof: Let $\Upsilon_{4}(\varsigma)$ be a Smarandache $T \mu \xi$ curve on the $\operatorname{surface} \psi(\varsigma, v)$. From (3) we find the parametric surface is defined by a given Smarandache $T \mu \xi$ as the subsequent:

$$
\psi(\varsigma, v)=\frac{1}{\sqrt{3}}(T(\varsigma)+\mu(\varsigma)+\xi(\varsigma))+[x(\varsigma, v) T(\varsigma)+y(\varsigma, v) \mu(\varsigma)+z(\varsigma, v) \xi(\varsigma)]
$$

Even since Smarandache curve of $T \mu \xi$ is an iso-parametric curve on the surface $\psi(\varsigma, v)$, there dwell a parameter $v=v_{0} \in\left[V_{1}, V_{2}\right]$ such that $\psi(\varsigma, v)=\frac{1}{\sqrt{3}}(T(\varsigma)+\mu(\varsigma)+\xi(\varsigma))$ and $\varsigma \in\left[L_{1}, L_{2}\right]$ then

$$
\mathrm{x}\left(\mathrm{s}, \mathrm{v}_{0}\right)=\mathrm{y}\left(\mathrm{s}, \mathrm{v}_{0}\right)=\mathrm{z}\left(\mathrm{s}, \mathrm{v}_{0}\right)=0
$$

From (4) the normal vector can be expressed as

$$
n\left(\varsigma, v_{0}\right)=\alpha\left(\varsigma, v_{0}\right) T(\varsigma)+\beta\left(\varsigma, v_{0}\right) \mu(\varsigma)+\gamma\left(\varsigma, v_{0}\right) \xi(\varsigma)
$$

Where 


$$
\left\{\begin{array}{c}
\alpha\left(\varsigma, \mathrm{v}_{0}\right)=\frac{\sqrt{3}}{3}\left(\mathrm{~K}_{\xi} \frac{\partial \mathrm{y}\left(\varsigma, \mathrm{v}_{0}\right)}{\partial \mathrm{v}}-\mathrm{K}_{\mu} \frac{\partial \mathrm{z}\left(\varsigma, \mathrm{v}_{0}\right)}{\partial \mathrm{v}}\right) \\
\beta\left(\varsigma, \mathrm{v}_{0}\right)=-\frac{\sqrt{3}}{3}\left[\mathrm{~K}_{\xi}\left(\frac{\partial \mathrm{x}\left(\varsigma, \mathrm{v}_{0}\right)}{\partial \mathrm{v}}+\frac{\partial \mathrm{z}\left(\varsigma, \mathrm{v}_{0}\right)}{\partial \mathrm{v}}\right)+\mathrm{K}_{\mu} \frac{\partial \mathrm{z}\left(\varsigma, \mathrm{v}_{0}\right)}{\partial \mathrm{v}}\right] \\
\gamma\left(\varsigma, \mathrm{v}_{0}\right)=\frac{\sqrt{3}}{3}\left[\mathrm{~K}_{\mu}\left(\frac{\partial \mathrm{x}\left(\varsigma, \mathrm{v}_{0}\right)}{\partial \mathrm{v}}+\frac{\partial \mathrm{y}\left(\varsigma, \mathrm{v}_{0}\right)}{\partial \mathrm{v}}\right)+\mathrm{K}_{\xi} \frac{\partial \mathrm{y}\left(\varsigma, \mathrm{v}_{0}\right)}{\partial \mathrm{v}}\right] .
\end{array}\right.
$$

From equation (1), (2) and (22) we find:

$$
n\left(\varsigma, v_{0}\right)=\Phi_{1}\left(\varsigma, v_{0}\right) T(\varsigma)+\Phi_{2}\left(\varsigma, v_{0}\right) N(\varsigma)+\Phi_{3}\left(\varsigma, v_{0}\right) B(\varsigma) .
$$

Where

$$
\left\{\begin{array}{c}
\Phi_{1}\left(\varsigma, \mathrm{v}_{0}\right)=\alpha\left(\varsigma, \mathrm{v}_{0}\right)=0, \\
\Phi_{2}\left(\varsigma, \mathrm{v}_{0}\right)=\beta\left(\varsigma, \mathrm{v}_{0}\right) \cos (\theta)-\gamma\left(\varsigma, \mathrm{v}_{0}\right) \sin (\theta) \neq 0, \\
\Phi_{3}\left(\varsigma, \mathrm{v}_{0}\right)=\beta\left(\varsigma, \mathrm{v}_{0}\right) \sin (\theta)+\gamma\left(\varsigma, \mathrm{v}_{0}\right) \cos (\theta)=0 .
\end{array}\right.
$$

From (22) and (23) we find $\Phi_{1}\left(\varsigma, v_{0}\right)=0$ and $\Phi_{3}\left(\varsigma, v_{0}\right)=0$ if and only if these the subsequent conditions are satisfied

$$
\left\{\begin{array}{c}
(1) \frac{\partial y\left(\varsigma, v_{0}\right)}{\partial v}=\frac{\mathrm{K}_{\mu}}{\mathrm{K}_{\xi}} \frac{\partial z\left(\varsigma, v_{0}\right)}{\partial v} \\
(2) \quad \mathrm{K}_{\xi}=\cot (\theta) \mathrm{K}_{\mu}
\end{array}\right.
$$

From $\Phi_{2} \neq 0$ and by using (24) we have

$$
\frac{-1}{\sqrt{3}} \mathrm{~K}_{\mu}\left\{\csc (\theta) \frac{\partial x\left(\varsigma, v_{0}\right)}{\partial v}+(\csc (\theta)+\sec (\theta)) \frac{\partial z\left(\varsigma, v_{0}\right)}{\partial v}\right\} \neq 0
$$

Then we find,

$$
\begin{gathered}
\frac{\partial x\left(\varsigma, v_{0}\right)}{\partial v} \neq-(1+\tan (\theta)) \frac{\partial z\left(\varsigma, v_{0}\right)}{\partial v} \\
\mathrm{~K}_{\mu} \neq 0,
\end{gathered}
$$

Then from (21), (24), (25) and (26) we have discovered the most important and adequate conditions for the $\psi\left(\zeta, v_{-} 0\right)$ have the spatial Smarandache $T \mu \xi$ curve of the curve is an iso-geodesic.

\section{Conclusion}

Now we found the necessary and su_cient conditions by using the Darboux frame that makes a special Smarandache curve is Iso-geodesic in Euclidean space, we need in the future to discuss whether it is possible or impossible to create the necessary conditions that make this curve is Iso-geodesic in Eculidean 7-space.

\section{Acknowledgment}

We wish to express our profound thanks and appreciation to the editor and the referees for their comments and suggestions to improve the paper.

\section{References}

C. Y. Li, R. H. Wang \& C.G. Zhu. (2011). Parametric representation of a surface pencil with a common line of curvature, Comput. Aided Des, 43(9), 1110-1117.https://doi.org/10.1016/j.cad.2011.05.001

F. Karakus \& Y. Yayli. (2016). The Fermi-Walker Derivative on the Spherical Indicatrix of Spacelike Curve in Minkowski 3-Space. Adv. Appl. Cli_. Algebr, 26, 625-644https://doi.org/10.1007/s00006-015-0635-9

G. J. Wang, K. Tang \& C. L. Tai (2004). Parametric representation of a surface pencil with a common spatial geodesic, Comput. Aided Des, 36(5), 447-459. https://doi.org/10.1016/S0010-4485(03)00117-9

L.N. Mishra (2017). On existence and behavior of solutions to some nonlinear integral equations with applications, Ph.D. Thesis, National Institute of Technology, Silchar 788 010, Assam, India.

O. Bektas. \& S. Yuce. (2013). Smarandache Curves According to Darboux Frame in Euclidean Space. Romanian Journal of Mathematics and Computer Science, 3(1), 48-59.

P. N. Azariadis \& N. A. Aspragathos. (2001). Geodesic curvature preservation in surface attening through constrained global optimization. Comput- Aided Des, 581-91.https://doi.org/10.1016/S0010-4485(00)00102-0

R. Brond, D. Jeulin, P. Gateau, J. Jarri \& G. Serpe. (1994). Estimation of the transport properties of polymer $\begin{array}{lllll}\text { composites by } & \text { geodesic }\end{array}$ 
167-77.https://doi.org/10.1111/j.1365-2818.1994.tb03511.x

R. J. Haw. (1985). An application of geodesic curves to sail design. Comput Graphics Forum, 4(2), 137-9.https://doi.org/10.1111/j.1467-8659.1985.tb00203.x

V. Caselles, R. Kimmel \& G. Sapiro. (1997). Geodesic active contours. Int J Comput Vision, 22(1), 61-79.https://doi.org/10.1023/A:1007979827043

\section{Copyrights}

Copyright for this article is retained by the author(s), with first publication rights granted to the journal.

This is an open-access article distributed under the terms and conditions of the Creative Commons Attribution license (http://creativecommons.org/licenses/by/4.0/). 